We re-analysed Cristea et al $\mathrm{s}^{3}$ effect sizes to demonstrate that, when procedures intended to modify cognitive bias elicit the process of cognitive bias modification, there is consistent impact on emotional disposition. Kruijt \& Carlbring contend that Cristea et als method of computing effect sizes compromises sensitivity to emotional disposition, which would represent a further limitation of this meta-analysis. However, compelling evidence that when procedures intended to evoke the process of cognitive bias modification do so successfully then so too do they alter emotional disposition, is not restricted to our re-analysis, and has been reported elsewhere. ${ }^{4}$

We advocate adherence to the experimental medicine framework, by clearly distinguishing two questions: one asks whether successfully modifying cognitive bias yields therapeutic benefit, and the other asks whether procedures intended to modify cognitive bias successfully induce this process of cognitive change.

Declaration of interest: B.G. is supported by Australian Research Council Grant DP170104533. C.M. is supported by Australian Research Council Grants DP170104533 and FL170100167. E.F. is supported by a European Research Council Advanced Investigator award (CogBIAS project REF: 324176 ).

1 Grafton B, MacLeod C, Rudaizky D, Holmes EA, Salemink E, Fox E, et al. Confusing procedures with process when appraising the impact of cognitive bias modification on emotional vulnerability. Br J Psychiatry 2017; 211: $266-71$.

2 Sheeran P, Klein WMP, Rothman AJ. Health behaviour change: moving from observation to intervention. Annu Rev Psychol 2017; 68: 573-600.

3 Cristea IA, Kok RN, Cuijpers P. Efficacy of cognitive bias modification interventions in anxiety and depression: meta-analysis. Br J Psychiatry 2015; 206 : 7-16.

4 Price RB, Wallace M, Kuckertz JM, Amir N, Graur S, Cummings L, et al. Pooled patient-level meta-analysis of children and adults completing a computerbased anxiety intervention targeting attentional bias. Clin Psychol Rev 2016; 50: 37-49.

Ben Grafton, PhD, Centre for the Advancement of Research on Emotion, School of Psychological Science, University of Western Australia, Crawley, Australia;

Colin MacLeod, PhD, Centre for the Advancement of Research on Emotion, School of Psychology, University of Western Australia, Crawley, Australia and School of Psychology, Babes-Bolyai University, Romania; Daniel Rudaizky, PhD, Centre for the Advancement of Research on Emotion, School of Psychology, University of Western Australia, Crawley, Australia; Elske Salemink, PhD, Developmental Psychology, University of Amsterdam, Amsterdam, the Netherlands; Elaine Fox, PhD, Oxford Centre for Emotion and Affective Neuroscience, and Department of Experimental Psychology, Research on Emotion, School of Psychology, University of Western Australia, Crawley, Australia. Email: ben.grafton@uwa.edu.au

doi:10.1192/bjp.2018.42

Author's reply: Kruijt \& Carlbring judiciously uncover significant methodological problems of the narrative re-analysis by Grafton and colleagues ${ }^{1}$ on our previous meta-analysis on the effectiveness of cognitive bias modification (CBM) interventions in anxiety and depression. ${ }^{2}$ The letter reinforces what we had previously noted in our invited comment, ${ }^{3}$ namely that our approach had been grossly misconstrued. In the meta-analysis, we had pooled all anxiety outcomes measured on validated instruments at post-intervention, whether these measured clinical symptoms, state or trait anxiety. We specifically excluded measures applied after a stressor induction task. If multiple measures in the same outcome category (for example general anxiety) were reported, we averaged them at study level. Grafton and colleagues claim to have re-analysed the anxiety data so as to reflect 'change in emotional vulnerability' (p. 268). Not only is this construct vague and its application susceptible to bias, but, as Kruijt \& Carlbring justly note, Grafton et al simply selected some of the already computed effect sizes and pooled them again. Essentially, this approach reflects the same mix comprising all anxiety outcomes, measured in the absence of a stressor induction task, and averaged at study level, just stemming from a more restricted pool of studies. To implement their new set of criteria, Grafton and colleagues should have recalculated effect sizes from study-level data, excluding measures and time points they did not deem appropriate for the elusive construct of emotional vulnerability. As it is, their re-analysis remains an arbitrary post hoc selection of study effects.

Yet a larger and more crucial problem relies in the central claim of Grafton et al, echoed by many leading CBM advocates: the effectiveness of these interventions should only be weighed if they successfully modified bias. Kruijt \& Carlbring adeptly liken this to familiar arguments for homeopathy. However, it also reflects a fundamental misunderstanding of how causal inferences and confounding function in a randomised design. Identifying the trials in which both bias and outcomes were successfully changed is only possible post hoc, as these are both outcomes measured after randomisation; reverse engineering the connection between the two is subject to confounding. Bias and symptom outcomes are usually measured at the same time points in the trial, thus making it impossible to establish temporal precedence. ${ }^{4}$ Circularity of effects, reverse causality (i.e. bias change causes symptom change or vice versa) and the distinct possibility of third variable effects (i.e. another variable causing both symptom and bias changes) further confound this relationship. ${ }^{4}$ For instance, trials where both bias and symptom outcomes were successfully modified could also be the ones with higher risk of bias, conducted by allegiant investigators, maximising demand characteristics or different in other, not immediately obvious, ways from trials where neither bias nor symptoms changed. Randomised controlled studies can only show whether an intervention to which participants were randomised has any effects on outcomes measured post-randomisation. ${ }^{5}$ Disentangling the precise components causally responsible for such effects is speculative and subject to confounding. To this point, randomised studies show CBM has a minute, unstable and mostly inexistent impact of any clinically relevant outcomes.

1 Grafton B, MacLeod C, Rudaizky D, Holmes EA, Salemink E, Fox E, et al. Confusing procedures with process when appraising the impact of cognitive bias modification on emotional vulnerability Br J Psychiatry 2017; 211: 266-71.

2 Cristea IA, Kok RN, Cuijpers P. Efficacy of cognitive bias modification interventions in anxiety and depression: meta-analysis. Br J Psychiatry 2015; 206 7-16.

3 Cristea IA, Kok RN, Cuijpers P. Invited commentary on ... Confusing procedures with process in cognitive bias modification research. Br J Psychiatry 2017; 211: 272-3.

4 Kazdin AE. Mediators and mechanisms of change in psychotherapy research. Ann Rev Clin Psychol 2007; 3: 1-27.

5 Kaptchuk TJ. The double-blind, randomized, placebo-controlled trial: gold standard or golden calf? J Clin Epidemiol 2001; 54: 541-9.

Ioana Alina Cristea, Department of Clinical Psychology and Psychotherapy, BabeșBolyai University, Cluj-Napoca, Romania and Department of Clinical, Neuro and Developmental Psychology, Amsterdam Public Health Research Institute, Vrije Universiteit Amsterdam, the Netherlands. Email: ioana.cristea@ubbcluj.ro

doi:10.1192/bjp.2018.43

\section{Towards a definition of unbearable suffering and the incongruence of psychiatric euthanasia}

In the article by Verhofstadt et al, the authors rightly observe that the concept of 'unbearable suffering' in relation to euthanasia remains poorly defined in the medical literature. ${ }^{1}$ We wish to make three observations which may contribute to a better understanding of 'unbearable suffering' and highlight the incongruence of considering euthanasia as psychotherapeutic. 